\title{
Diagnosis and pathologic characteristics of medullary thyroid carcinoma-review of current guidelines
}

\author{
C.M. Thomas MD PhD, ${ }^{* \dagger}$ S.L. Asa MD PhD, ${ }^{\ddagger \S}$ S. Ezzat MD, I A.M. Sawka MD PhD, ${ }^{\#}$ and D. Goldstein $\mathrm{MD}^{* \dagger}$
}

\section{ABSTRACT}

Background Medullary thyroid carcinoma (MTC) is a rare malignancy of the thyroid gland, and raising awareness of the recommended diagnostic workup and pathologic characteristics of this malignancy is therefore important.

Methods We reviewed the current clinical practice guidelines and recent literature on мTс, and here, we summarize the recommendations for its diagnosis and workup. We also provide an overview of the pathology of мTc.

Results A neuroendocrine tumour, MTC arises from parafollicular cells (“C cells"), which secrete calcitonin. As part of the multiple endocrine neoplasia (MEN) type 2 syndromes, MTC can occur sporadically or in a hereditary form. This usually poorly delineated and infiltrative tumour is composed of solid nests of discohesive cells within a fibrous stroma that might also contain amyloid. Suspicious nodules on thyroid ultrasonography should be assessed with fine-needle aspiration (FNA). If a diagnosis of MTC is made on FNA, patients require baseline measurements of serum calcitonin and carcinoembryonic antigen. Calcitonin levels greater than $500 \mathrm{pg} / \mathrm{mL}$ or clinical suspicion for metastatic disease dictate the need for further imaging studies. All patients should undergo DNA analysis for RET mutations to diagnose MEN type 2 syndromes, and if positive, they should be assessed for possible pheochromocytoma and hyperparathyroidism.

Summary Although the initial diagnosis of a suspicious thyroid nodule is the same for differentiated thyroid carcinoma and MTC, the remainder of the workup and diagnosis for MTC is distinct.

Key Words Medullary thyroid carcinoma, MEN2, diagnosis, calcitonin, carcinoembryonic antigen, RET, amyloid, hyperparathyroidism, pheochromocytoma

Curr Oncol. 2019 October;26(5):338-344

www.current-oncology.com

\section{INTRODUCTION}

In the general population, the prevalence of palpable thyroid nodules ranges from $1 \%$ (in male subjects) to $5 \%$ (in female subjects), and the detection of thyroid nodules by ultrasonography ranges from $19 \%$ to $68 \%$, again higher in female and elderly patients ${ }^{1}$. The incidence of thyroid cancer in the United States is 7.7 per 100,000, which has been slowly increasing, likely because of increased detection of thyroid nodules ${ }^{2,3}$. When evaluating a solitary thyroid nodule, suspicion for malignancy is increased by extremes of age; male sex; family history of thyroid cancer; history of radiation exposure; and physical exam findings, including a firm, fixed nodule and vocal cord paralysis ${ }^{4}$. Almost all of these malignancies $(>90 \%)$ are differentiated thyroid carcinoma (DTC), including papillary (PTC) and follicular carcinoma, with a much smaller percentage being medullary and anaplastic thyroid carcinomas ${ }^{5}$. Historically, medullary thyroid carcinoma (MTC) accounted for $5 \%-8 \%$ of all thyroid malignancies ${ }^{2}$, but a recent analysis of the U.S. Surveillance, Epidemiology, and End Results program database revealed a lower rate of $1 \%-2 \%$ of all thyroid cancers in the United States ${ }^{6}$. The incidence of PTC is

Sanofi Genzyme supported the publication of this series with an unrestricted educational grant. They had no influence over the content. 
5.7 per 100,000 ; the incidence of MTC is 0.11 per $100,000^{2}$. Most clinicians, even those in a busy thyroid practice, have significantly less experience with diagnosing, managing, treating, and knowing the pathologic characteristics of MTC compared with more common thyroid neoplasms. It is therefore pertinent to heighten awareness of this relatively rare malignancy.

Hazard et al. ${ }^{7}$ published the first histologic description of MTC in 1959. A neuroendocrine tumour, MTC arises from parafollicular cells ("C cells"), which are of neural crest origin and secrete calcitonin ${ }^{8-10}$. Medullary thyroid carcinoma can occur sporadically ( $75 \%$ of cases) or in a hereditary form (25\% of cases) as part of the multiple endocrine neoplasia (MEN) type 2 syndromes MEN2A and MEN2B, which are autosomal-dominant. The RET proto-oncogene encodes a transmembrane receptor of the tyrosine kinase family and exhibits germline mutation in patients with MEN2A and MEN2B, and somatic mutation in $50 \%$ of sporadic MTCs $^{11-14}$. Within the MEN type 2 syndromes, MEN2A is more common (95\%) and consists of 4 variants: classical MEN2A with pheochromocytoma and hyperparathyroidism, MEN2A with cutaneous lichen amyloidosis, MEN2A with Hirschsprung disease, and familial MTC. In the remaining $5 \%$ of cases (MEN2B), patients have мтс, pheochromocytoma, and a distinctive appearance $^{6}$. A negative family history does not exclude germline disease, because generations can be skipped, de novo mutations can arise, or MTC can be inherited without the other endocrinopathies ${ }^{15}$. Sporadic MTc typically presents in the 5th or 6th decade of life; hereditary forms generally present earlier ${ }^{16}$.

The goals of the present narrative review were to examine the current clinical practice guidelines and recent literature about MTC and to concisely summarize the recommendations for diagnosis and workup of MTC as well as the pathology findings. The American Thyroid Association (ATA) published guidelines for the management of MTC in 2009 , with an update in $2015^{6}$. Other groups that have published guidelines on MTC include the European Society for Medical Oncology, the U.S. National Comprehensive Cancer Network, and the British Thyroid Foundation, and consensus documents have emerged from international workshops on $\mathrm{MEN}^{2,15-17}$.

Our review of MTC diagnosis encompasses fine-needle aspiration (FNA) of suspicious nodules; measurement of serum calcitonin and carcinoembryonic antigen (CEA); germline RET genetic testing, and if positive, the workup for associated endocrinopathies; and evaluation for distant metastases. In addition, the pathology of MTC is reviewed. Our review is not intended to replace individual physician decision-making, but to serve as a narrative summary of the most recent literature, guidelines, and recommendations on мTс from other organizations. The article by Larcouche et al. ${ }^{18}$ in the next issue of Current Oncology covers the genetic aspects of this disease group in more detail.

\section{DISCUSSION}

In MTC, management should be performed by a multidisciplinary team consisting of an otolaryngologist or endocrine surgeon, medical oncologist, endocrinologist, and pathologist with support from multiple other specialties.

\section{Evaluation of a Thyroid Nodule}

The ATA management guideline for adult patients with thyroid nodules was updated in $2015^{19}$. The guideline provides the best available evidence for the diagnosis and treatment of thyroid nodules.

A thyroid nodule is defined as a distinct lesion within the thyroid gland that is radiographically different from the surrounding tissue. The initial evaluation of a thyroid nodule must include ultrasonography assessment, because certain sonographic patterns are associated with varying risks of malignancy and, in addition to nodule size, drive the decision for FNA ${ }^{20,21}$. High-suspicion nodules-those that are hypoechoic with irregular borders, microcalcifications, taller-than-wide morphology, or obvious extrathyroidal extension-have a risk of malignancy of $70 \%-90 \%$. Hypoechoic nodules that are solid with a regular border are intermediate-suspicion nodules with a malignancy risk of $10 \%-20 \%^{19}$. The ATA makes a strong recommendation for FNA of nodules $1 \mathrm{~cm}$ or greater in size that have a highsuspicion (moderate-quality evidence) or intermediatesuspicion (low-quality evidence) sonographic pattern ${ }^{19}$.

Despite the differences in the biologic behaviors of DTC and MTC, the decision for when to perform a FNA of a thyroid nodule is similar. Literature about ultrasound features suggestive of MTC is limited because of the relative rarity of the disease. Most published studies demonstrate that the ultrasound criteria for MTC are not significantly different from the much more common DTC, with the exception of a higher prevalence of round or oval shapes in MTC $^{22,23}$. In one study of 30 patients with MTC (36 nodules), the characteristic ultrasound findings were hypoechoic ( $n=26,72.2 \%)$, solid $(n=33,91.7 \%)$, smooth borders $(n=19$, $52.8 \%)$, round or oval shape $(n=23,63.9 \%)$, and micro- or macrocalcifications $(n=21,58.3 \%)^{22}$. Increased vascularity has also been seen in MTc $(90.9 \%, n=40)$ compared with PTC $(9.5 \%, n=47)^{23}$. In a study by Trimboli et al. ${ }^{24}$, the sonographic features of MTC (12 patients), PTC (39 patients), and benign nodules (254 patients) were compared. The rate of hypoechogenicity was $69.2 \%$ for PTC ( $n=27), 50 \%$ for мтс $(n=6)$, and $20.1 \%$ for benign nodules $(n=51), p<0.001$. Most benign and MTc nodules had regular borders $(63.4 \%$, $n=161$, and $75 \%, n=9$, respectively); most PTc nodules had irregular borders $(64.1 \%, n=25)$. Finally, although microcalcifications were more common in MTc than in benign nodules $(16.7 \%, n=2$, vs. $0.8 \%, n=2)$, they are significantly more common in PTC $(69.2 \%, n=27)^{24}$. Adhering to the ATA guidelines for FNA of thyroid nodules based on ultrasound characteristics for DTC should identify most cases of MTC. However, the fact that the sonographic features of мтс are sometimes benign constitute one argument for measuring serum calcitonin, as recommended by some groups, in all patients presenting with a thyroid nodule (discussed later in this article $)^{25}$.

\section{Diagnosis of MTC on FNA}

The cytologic appearance of MTC on FNA can be variable, causing MTC to be misdiagnosed as a follicular neoplasm, a sarcoma, or a plasmacytoma ${ }^{6}$, and the accuracy of FNA has been variably reported in the literature. Some individual studies examining the diagnostic accuracy of FNA for MTC have shown that it is a good diagnostic method. For 
example, Chang et al. ${ }^{26}$ examined the cytology from FNAs in 34 cases of MTC and found that the diagnosis was correct in $82.4 \%$ of the cases $(n=28)$, and the samples were suspicious for MTC in $5.9 \%(n=2)$. Only 3 cases $(8.8 \%)$ were misdiagnosed. Another study found that $89.0 \%$ of MTC cases (81 of 91) were diagnosed correctly on cytology ${ }^{27}$. In contrast, however, a meta-analysis of fifteen studies involving 641 cases of FNA for MTC demonstrated a detection rate of only $56.4 \%$ on cytology [ $95 \%$ confidence interval (CI): $52.6 \%$ to $60.1 \%]^{28}$.

A more accurate method of diagnosing MTc is to measure calcitonin in the aspirate specimen. A meta-analysis of twelve studies involving 413 thyroid nodules of varying pathology demonstrated that FNA calcitonin diagnosed 93 of the 95 nodules that were MTC $(97.9 \%)^{29}$. As demonstrated in a study by Trimboli et al. ${ }^{30}$, FNA calcitonin is more sensitive than cytology for diagnosing MTc. In their study, FNA calcitonin was obtained in 38 mTC lesions (34 thyroid nodules, 4 lymph nodes) and in 52 control thyroid nodules ${ }^{30}$. Compared with cytology alone, which had a sensitivity of $58.8 \%$ ( $95 \%$ Cr: $40.7 \%$ to $75.3 \%$ ), the use of FNA calcitonin to detect MTC had a sensitivity of $100 \%$ (95\% CI: $89.6 \%$ to $100 \%)^{30}$. When the study used a threshold value of $39.6 \mathrm{pg} / \mathrm{mL}$ for FNA calcitonin, accuracy was $100 \%{ }^{30}$. A wide range of FNA calcitonin threshold values have been reported in the literature $(7.4-67 \mathrm{pg} / \mathrm{mL})^{28}$. Immunohistochemistry staining of FNA specimens for calcitonin, CEA, and chromogranin can also be performed ${ }^{6}$, increasing the sensitivity of cytology to $89.2 \%$ (95\% CI: $74.6 \%$ to $96.9 \%)^{30}$.

The revised ATA guideline for MTC now recommends measurement of calcitonin in FNA specimens and immunohistochemistry for calcitonin, CEA, and chromogranin when cytology is inconclusive or suggestive of MTC (grade B recommendation based on fair evidence); however, the guideline does not recommend a threshold value for calcitonin ${ }^{6}$. Despite such testing, a significant failure to make a correct diagnosis could still occur in almost $44 \%$ of cases because the initial suspicion for MTC on cytology is not there. Once MTC is diagnosed on FNA, measurement of serum calcitonin and CEA, analysis for a RET germline mutation, and a staging workup should follow.

\section{Testing for Secretory Products of MTC}

Secretion of calcitonin and CEA by MTC occurs in direct proportion to the $\mathrm{C}$ cell mass. Although measurement of serum calcitonin in all patients with a thyroid nodule is not universally endorsed or practiced, some groups recommend it ${ }^{16}$. Elevated serum calcitonin might help to identify thyroid nodules that have benign features on ultrasound and yet harbour MTC. Controversy attends that recommendation, with the ATA remaining equivocal and the U.S. National Comprehensive Cancer Network recommending against routine measurement of serum calcitonin in all patients with thyroid nodules ${ }^{16}$.

Baseline calcitonin levels of $60-100 \mathrm{pg} / \mathrm{mL}$ are highly indicative of MTC, but the clinical implications of lower concentrations are unclear ${ }^{15}$. Serum calcitonin can be altered by medications such as proton pump inhibitors, renal failure, hypercalcemia, PTC, lymphocytic thyroiditis, hypothyroidism, and pregnancy ${ }^{15}$. Serum calcitonin does not increase in response to calcium or pentagastrin stimulation (which has limited availability) in nonthyroid malignancies and other disease processes as it does in $\mathrm{MTC}^{31}$. In the past, stimulation tests were used in the diagnosis of MTC, especially when calcitonin was mildly elevated. However, with the more sensitive calcitonin assays currently available, stimulation testing is not routinely recommended ${ }^{15}$. The low incidence of MTC makes measurement of serum calcitonin in all patients with a thyroid nodule not cost-effective and is one reason for the lack of consensus about whether it should be performed ${ }^{16}$. In MTC, CEA is not a specific biomarker, but it is useful for monitoring disease progression ${ }^{32}$. Although measuring calcitonin in all patients with a thyroid nodule is controversial, baseline levels of both calcitonin and CEA should be measured when a diagnosis of MTC has been made. Normal or low levels of both serum calcitonin and CEA, or a markedly elevated cEA level out of proportion to calcitonin can indicate poorly differentiated MTc. In addition, baseline levels of calcitonin can indicate distant metastases-such metastases being unlikely if baseline serum calcitonin is less than $500 \mathrm{pg} / \mathrm{mL}^{2,33}$.

\section{Analysis for RET Germline Mutation}

A DNA analysis must be done to assess for RET germline mutations, and if such mutations are present, patients require an evaluation for pheochromocytoma and hyperparathyroidism. Specific RET mutations are associated with disease aggressiveness and dictate early timing of thyroidectomy ${ }^{6,16,34}$. The order of mutation testing is based on clinical features and the level of suspicion for MEN2A or MEN2B. In the absence of clinical features, RET mutations in exons $10-11$ are tested first, and if negative, then in exons $13-16^{15,17}$. Genetic testing and appropriate counselling must be offered to first-degree relatives in addition to the patient.

In MEN2A, the rate of unilateral or bilateral pheochromocytoma is $50 \%$, and the rate of hyperparathyroidism is $20 \%-30 \%{ }^{17}$. Evaluation for pheochromocytoma includes measurements of plasma metanephrines and normetanephrines or a 24-hour urine analysis for catecholamines or metanephrines and normetanephrines ${ }^{2,15}$. Before MTC is treated, diagnosis of a pheochromocytoma is essential to avoid a hypertensive crisis during surgery ${ }^{16}$. Workup for hyperparathyroidism includes measurement of serum calcium and parathyroid hormone ${ }^{16}$. Identification of hyperparathyroidism in the preoperative period allows for appropriate planning to address both the MTC and the parathyroid glands during the same operative procedure. Some recommendations, such as those proposed by the British Thyroid Association, state that testing for pheochromocytoma and hyperparathyroidism should occur in all patients with MTc regardless of symptoms, family history, or RET mutation status ${ }^{15}$.

\section{Recommendations for Preoperative Imaging}

Distant metastases are more likely if serum calcitonin is greater than $500 \mathrm{pg} / \mathrm{mL}$, and further imaging is recommended. However, systemic imaging is recommended regardless of calcitonin level if neck disease is extensive or in the presence of other concerns for distant metastases. Approximately $50 \%$ of patients with MTC will have metastatic disease on initial presentation ${ }^{2}$. In sporadic MTC, the 
rates of central and lateral neck lymph node metastases associated with $\mathrm{T} 1$ tumours are $14 \%$ and $11 \%$ respectively; the rates are $86 \%$ and $93 \%$ respectively with pT4 tumours ${ }^{35}$. Palpable thyroid nodules are associated with a $70 \%$ rate of lymph node metastasis and a $10 \%$ rate of distant metastasis $^{36}$. Recommended imaging studies include neck ultrasonography, computed tomography (Ст) of lungs and mediastinum, 3-phase contrast-enhanced multi-detector liver Ст or contrast-enhanced magnetic resonance imaging of liver, and magnetic resonance imaging or bone scintigraphy ${ }^{6}$. Integrated ${ }^{18} \mathrm{~F}$-fluorodeoxyglucose positron-emission tomography and ст is less sensitive in detecting metastases and is therefore not recommended ${ }^{15,37}$.

\section{Pathology Diagnosis}

The diagnosis of MTC is often made at surgical pathology after the lobe or thyroid is resected. These tumours can have a characteristic morphology, demonstrating the solid and nesting growth pattern of neuroendocrine tumours in many sites. Some form conspicuous amyloid (a deposition of pre-procalcitonin) that is highly characteristic (Figure 1). However, they can have unusual morphologies ${ }^{38}$ that require immunohistochemistry for confirmation. Pseudopapillary variants mimic PTC; they might infiltrate around follicles or form proteinaceous material that can mimic follicles, resembling follicular neoplasms. Oncocytic variants are misdiagnosed as Hürthle cell carcinoma. Plasmacytoid variants can be mistaken for plasmacytoma. Spindle-cell and giant-cell variants can be mistaken for sarcomas or anaplastic carcinomas, and rare but aggressive small-cell types resemble lymphomas. The diagnosis should be confirmed with immunostains for chromogranin, calcitonin, and CEA, which all serve as biomarkers for postoperative surveillance.

In other neuroendocrine tumours, $\mathrm{Ki}-67$ is used for grading, but the application of that modality for MTC remains unclear ${ }^{39}$. The tumours should be appropriately staged using the synoptic cancer worksheets proposed by the College of American Pathologists ${ }^{40}$. Multifocality or $\mathrm{C}$ cell hyperplasia in the contralateral lobe should be assessed, because those features indicate a strong likelihood of germline RET mutation and inherited disease ${ }^{38}$.

\section{SUMMARY}

Medullary thyroid carcinoma is usually a poorly delineated and infiltrative tumour composed of solid nests of discohesive cells within a fibrous stroma that might also contain amyloid. Tumour cells stain for calcitonin, chromogranin A, and CEA. The diagnosis can be missed when MTCs have a follicular architecture, a pseudopapillary pattern, or an oncocytic appearance, and they can be misclassified as follicular cell-derived lesions. The initial diagnosis of a suspicious thyroid nodule is the same in DTC and мTC, but the remainder of the workup for a diagnosis of MTC is distinct. After a diagnosis of MTC on FNA, next steps should be measurement of serum calcitonin and CEA, analysis for a RET germline mutation, the appropriate workup for pheochromocytoma and hyperparathyroidism as indicated based on RET mutation status, and assessment for metastatic disease.

\section{CONFLICT OF INTEREST DISCLOSURES}

We have read and understood Current Oncology's policy on disclosing conflicts of interest, and we declare that we have none.

\section{AUTHOR AFFILIATIONS}

*Department of Otolaryngology-Head and Neck Surgery and ${ }^{\dagger}$ Department of Surgical Oncology, Princess Margaret Cancer Centre, University Health Network, Toronto, ON; ${ }^{\ddagger}$ Department of Pathology, Case Western Reserve University, Cleveland, OH, U.S.A.; ${ }^{\S}$ Department of Pathology, University Health Network, "Department of Medicine and Oncology, University of Toronto,
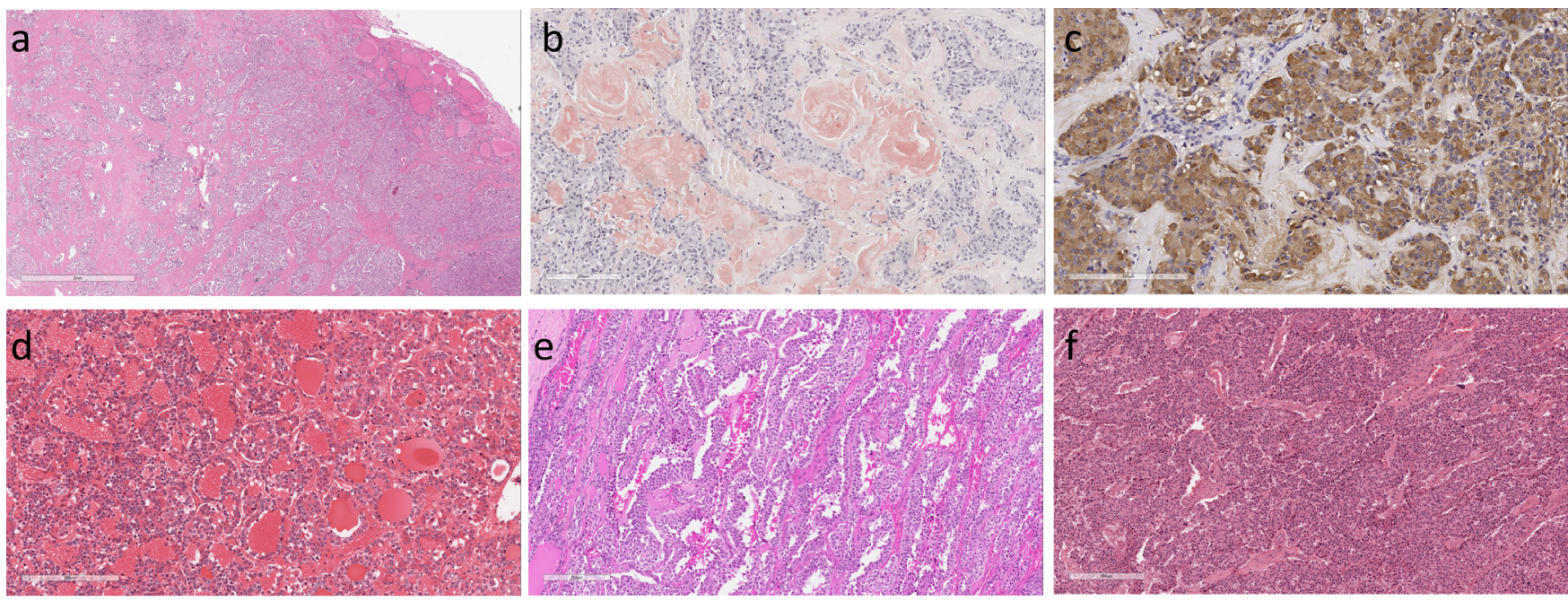

FIGURE 1 Pathology of medullary thyroid carcinoma. This usually poorly delineated and infiltrative tumour is composed of solid nests of discohesive cells within a fibrous stroma that might also contain amyloid. (a) The amyloid can be recognized by its intense staining with Congo red. (b) It also exhibits apple green birefringence with polarized light (not shown). (c) Tumour cells stain for calcitonin as well as for chromogranin A and carcinoembryonic antigen (not shown). The diagnosis can be missed when a medullary thyroid carcinoma (d) has a follicular architecture, (e) pseudopapillary patterns, or $(\mathrm{f})$ is oncocytic, and they can be misclassified as follicular cell-derived lesions. 
and ${ }^{\#}$ Department of Medicine, Division of Endocrinology, University of Toronto, Toronto, ON.

\section{REFERENCES}

1. Dean DS, Gharib H. Epidemiology of thyroid nodules. Best Pract Res Clin Endocrinol Metab 2008;22:901-11.

2. Pacini F, Castagna MG, Brilli L, Pentheroudakis G on behalf of the Esmo Guidelines Working Group. Thyroid cancer: ESMO clinical practice guidelines for diagnosis, treatment and follow-up. Ann Oncol 2012;23 (suppl 7):vii110-19.

3. British Thyroid Association. Guidelines for the Management of Thyroid Cancer. Report of the Thyroid Cancer Guidelines Update Group. 2nd ed. London, U.K.: Royal College of Physicians; 2007.

4. Hegedus L. Clinical practice. The thyroid nodule. $N$ Engl J Med 2004;351:1764-71.

5. Sherman SI. Thyroid carcinoma. Lancet 2003;361:501-11.

6. Wells SA Jr, Asa SL, Dralle H, et al. on behalf of the American Thyroid Association Guidelines Task Force on Medullary Thyroid Carcinoma. Revised American Thyroid Association guidelines for the management of medullary thyroid carcinoma. Thyroid 2015;25:567-610.

7. Hazard JB, Hawk WA, Crile G Jr. Medullary (solid) carcinoma of the thyroid; a clinicopathologic entity. J Clin Endocrinol Metab 1959;19:152-61.

8. Tashjian AH Jr, Melvin EW. Medullary carcinoma of the thyroid gland. Studies of thyrocalcitonin in plasma and tumor extracts. N Engl J Med 1968;279:279-83.

9. Wells SA Jr, Baylin SB, Linehan WM, Farrell RE, Cox EB, Cooper CW. Provocative agents and the diagnosis of medullary carcinoma of the thyroid gland. Ann Surg 1978;188:139-41.

10. Williams ED. Histogenesis of medullary carcinoma of the thyroid. J Clin Pathol 1966;19:114-18.

11. Carlson KM, Dou S, Chi D, et al. Single missense mutation in the tyrosine kinase catalytic domain of the RET protooncogene is associated with multiple endocrine neoplasia type 2B. Proc Natl Acad Sci U S A 1994;91:1579-83.

12. Hofstra RM, Landsvater RM, Ceccherini I, et al. A mutation in the RET proto-oncogene associated with multiple endocrine neoplasia type $2 \mathrm{~B}$ and sporadic medullary thyroid carcinoma. Nature 1994;367:375-6.

13. Marsh DJ, Learoyd DL, Andrew SD, et al. Somatic mutations in the RET proto-oncogene in sporadic medullary thyroid carcinoma. Clin Endocrinol (Oxf) 1996;44:249-57.

14. Mulligan LM, KwokJB, Healey CS, et al. Germ-line mutations of the RET proto-oncogene in multiple endocrine neoplasia type 2A. Nature 1993;363:458-60.

15. Perros P, Boelaert K, Colley S, et al. on behalf of the British Thyroid Association. Guidelines for the management of thyroid cancer. Clin Endocrinol (Oxf) 2014;81(suppl 1):1-122.

16. Tuttle RM, Ball DW, Byrd D, et al. on behalf of the National Comprehensive Cancer Network. Medullary carcinoma. $J$ Natl Compr Canc Netw 2010;8:512-30.

17. Brandi ML, Gagel RF, Angeli A, et al. Guidelines for diagnosis and therapy of Men type 1 and type 2.JClin Endocrinol Metab 2001;86:5658-71.

18. Larouche V, Akirov A, Thomas CM, Krzyzanowska MK, Ezzat Shereen. A primer on the genetics of medullary thyroid cancer. Curr Oncol 2019;26:[in press].

19. Haugen BR, Alexander EK, Bible KC, et al.2015American Thyroid Association management guidelines for adult patients with thyroid nodules and differentiated thyroid cancer: the American Thyroid Association Guidelines Task Force on thyroid nodules and differentiated thyroid cancer. Thyroid 2016;26:1-133.

20. Brito JP, Gionfriddo MR, Al Nofal A, et al. The accuracy of thyroid nodule ultrasound to predict thyroid cancer: systematic review and meta-analysis. J Clin Endocrinol Metab 2014;99:1253-63.
21. Smith-Bindman R, Lebda P, Feldstein VA, et al. Risk of thyroid cancer based on thyroid ultrasound imaging characteristics: results of a population-based study. JAMA Intern Med 2013;173:1788-96.

22. Choi N, Moon WJ, Lee JH, BaekJH, Kim DW, Park SW. Ultrasonographic findings of medullary thyroid cancer: differences according to tumor size and correlation with fine needle aspiration results. Acta Radiol 2011;52:312-16.

23. Liu MJ, Liu ZF, Hou YY, et al. Ultrasonographic characteristics of medullary thyroid carcinoma: a comparison with papillary thyroid carcinoma. Oncotarget 2017;8:27520-8.

24. Trimboli P, Nasrollah N, Amendola S, et al. Should we use ultrasound features associated with papillary thyroid cancer in diagnosing medullary thyroid cancer? Endocr $J$ 2012;59:503-8.

25. Trimboli P, Giovanella L, Valabrega S, et al. Ultrasound features of medullary thyroid carcinoma correlate with cancer aggressiveness: a retrospective multicenter study. J Exp Clin Cancer Res 2014;33:87.

26. Chang TC, Wu SL, Hsiao YL. Medullary thyroid carcinoma: pitfalls in diagnosis by fine needle aspiration cytology and relationship of cytomorphology to $R E T$ proto-oncogene mutations. Acta Cytol 2005;49:477-82.

27. Papaparaskeva K, Nagel H, Droese M. Cytologic diagnosis of medullary carcinoma of the thyroid gland. Diagn Cytopathol 2000;22:351-8.

28. Trimboli P, Treglia G, Guidobaldi L, et al. Detection rate of FNA cytology in medullary thyroid carcinoma: a meta-analysis. Clin Endocrinol (Oxf) 2015;82:280-5.

29. Trimboli P, Guidobaldi L, Bongiovanni M, Crescenzi A, Alevizaki M, Giovanella L. Use of fine-needle aspirate calcitonin to detect medullary thyroid carcinoma: a systematic review. Diagn Cytopathol 2016;44:45-51.

30. Trimboli P, Cremonini N, Ceriani L, et al. Calcitonin measurement in aspiration needle washout fluids has higher sensitivity than cytology in detecting medullary thyroid cancer: a retrospective multicentre study. Clin Endocrinol (Oxf) 2014;80:135-40.

31. Toledo SP, Lourenco DM Jr, Santos MA, Tavares MR, Toledo RA, Correia-Deur JE. Hypercalcitoninemia is not pathognomonic of medullary thyroid carcinoma. Clinics (Sao Paulo) 2009;64:699-706.

32. Wells SA Jr, Haagensen DE Jr, Linehan WM, Farrell RE, Dilley WG. The detection of elevated plasma levels of carcinoembryonic antigen in patients with suspected or established medullary thyroid carcinoma. Cancer 1978;42(suppl):1498-503.

33. Machens A, Dralle H. Biomarker-based risk stratification for previously untreated medullary thyroid cancer. J Clin Endocrinol Metab 2010;95:2655-63.

34. Chen H, Sippel RS, O'Dorisio MS, Vinik AI, Lloyd RV, Pacak K on behalf of the North American Neuroendocrine Tumor Society. The North American Neuroendocrine Tumor Society consensus guideline for the diagnosis and management of neuroendocrine tumors: pheochromocytoma, paraganglioma, and medullary thyroid cancer. Pancreas 2010;39:775-83.

35. Machens A, Hinze R, Thomusch O, Dralle H. Pattern of nodal metastasis for primary and reoperative thyroid cancer. World J Surg 2002;26:22-8.

36. Moley JF. Medullary thyroid carcinoma: management of lymph node metastases. J Natl Compr Canc Netw 2010;8:549-56.

37. Giraudet AL, Vanel D, Leboulleux S, et al. Imaging medullary thyroid carcinoma with persistent elevated calcitonin levels. J Clin Endocrinol Metab 2007;92:4185-90.

38. Boerner SL AS. Biopsy Interpretation of the Thyroid. 2nd ed. Philadelphia, PA: Wolters Kluwer; 2017. 
39. Rindi G, Klimstra DS, Abedi-Ardekani B, et al. A common classification framework for neuroendocrine neoplasms: an International Agency for Research on Cancer (IARC) and World Health Organization (wHo) expert consensus proposal. Mod Pathol 2018;31:1770-86.
40. College of American Pathologists (CAP). Cancer Protocol Templates [Web page]. Northfield, IL: CAP; 2019. [Available at: https://www.cap.org/protocols-and-guidelines/cancerreporting-tools/cancer-protocol-templates; cited 16 June 2019] 